\title{
Asymmetric molecular diode energy calculation using Extended Hückel and Parametric method
}

\author{
A. Mallaiah ${ }^{1}$, G. N. Swamy ${ }^{2}$, K. Padmapriya ${ }^{3}$ \\ ${ }^{1}$ Research Scholar, JNTUA, Anthapuramu, A.P, India \\ ${ }^{2}$ Department of EI\&E, VR Siddhartha Engineering College, Vijayawada, A.P, India \\ ${ }^{3}$ Department of ECE, JNTUK, Kakinada, A.P, India \\ malli797@gmail.com
}

PACS 31.15.bu, 73.40.Ei, 73.40.Cg, 81.07.Nb

DOI 10.17586/2220-8054-2016-7-3-569-574

\begin{abstract}
The Electrical rectification properties of an asymmetric molecule's amine group and nitro group has been studied by placing the compound between two gold electrodes and using Extended Hückel, Parametric and non-equilibrium Green's function (NEGF) formalisms. The conductance of the device falls exponentially with an increased number of $\mathrm{CH}_{2}$ moieties in the molecule. Current rectification was observed based on HOMO, LUMO gaps and potential drop across the molecules. The investigation of the spatial dispersion of frontier orbitals, the highest occupied molecular orbitals, lowest unoccupied molecular (HOMO-LUMO) of the molecule command the transmission of electrons in the molecule. The results demonstrate that, depending on the group of molecules and number of $\mathrm{CH}_{2}$ moieties present, current shipping from left side of device to right side of device based on orbital energy gaps. Our findings demonstrate that a true molecular diode can be created, and thus miniaturize the electronic circuit's size to the Nano scale.
\end{abstract}

Keywords: HOMO, LUMO, orbital energy, molecular diode, NEGF.

Received: 18 February 2016. Revised: 11 May 2016. Final revision: 13 May 2016

\section{Introduction}

One of the most active fields in nanotechnology research is the fabrication of electronic circuits with Nano scale devices. Several research groups have been succeeded in fabricating Nano devices in form of rectifiers, switches, logic gates and flip flops in which active part of device is single molecule and metallic Nano wire [9].

Aviram and Ratner [1] suggested that a single molecule with a Donor bridge Acceptor (DBA) behaves as a diode when placed in an electric field. Electrons can move cathode terminal to acceptor, and electrons from donor and then transferred towards anode. The working principle of this device is similar to "valve" diode introduced by Shockley 65 years back. Such hybrid molecular electronic (HME) device comprising molecules embedded between electrodes of utilizing donor molecules $\mathrm{NH}_{2}, \mathrm{OH}, \mathrm{CH}_{3}, \mathrm{CH}_{2} \mathrm{CH}_{3}$, acceptor molecules $\mathrm{NO}_{2}, \mathrm{CN}, \mathrm{CHO}, \mathrm{COR}$ ' where R' is an aliphatic chain [4].

The source of conductivity for a poly phenylene based chains is a set of $\pi$ type orbitals that lie supra and infra the level of the molecule when it is of a planar or nearly planar geometry. In a planar conformation the $\pi$ orbitals associated with each individual atom convergence or coupled in various combinations to create a set of extended $\pi$ orbitals that crosses the length of the molecule. This occurs because there is a significant energetic advantage that arises from delocalizing valance electrons in orbitals that span or nearly span the length of the entire molecule. The low energy $\pi$ orbital available in the molecule and high energy $\pi$ orbital dissent somewhat they do have nodal planes (planes where the orbital vanish) pointed perpendicularly to the axis of a wire like molecule. The higher the energy of the orbital the more nodal planes it will contain [15].

A delocalized $\pi$ orbital usually extended across one or more of the neighboring aromatic rings in the molecule as well as across other intervene multiply bonded groups can add or merge to form a number of larger moleculespanning $\pi$ orbitals, each molecule bearing [4] a different nodal structure and energy. There are five possible asymmetric factors involved in the junction: electrode material; electrode shape; interface [6]; configuration of junction; and the nature of the molecular bridge. In this paper our discussion concerns the molecular bridge. Like semiconducting material the introduction of electron donating and electron receiving groups into the molecular wire can achieve the $\mathrm{p}-\mathrm{n}$ junction like molecules. These materials have been exploited as the key electronic components with a light emitting diodes, transistors, sensor devices, photovoltaic solar cells.

Recently Nonequlibrium green's function (NEGF) formalism has become a powerful tool to explore electronic transportation [14] at a real molecular level due to the contribution by Datta and co-workers [7-9], 
Ratner and co-workers [23-25], Di Carlo and co-workers [12], Xue and co-workers [10], Guo and coworkers [13], and so on. Although it has been used in a wide variety of systems, there is less concern on the effect of asymmetrically substituent groups in previous work. In the present work we will consider a series of model systems constructed by two sulfur atoms by a substituted molecule as shown in Fig. 1 and exploit the potential molecular rectification.

This paper organized as follows: In section 2 we give a brief description of the calculation method and structure of molecules. Section 3 shows the detailed analysis of the HOMO \& LUMO energies between the sulfur atoms. The effect of the molecular orbitals and energies are analyzed. The HOMO, LUMO gap (HLG) and potential drop listed. A brief summary is given in Section 4.

\section{Computational methods}

\subsection{Geometry optimization}

We performed geometry optimization of the DBA diode nanowire. Our model includes sulfur atoms attached on each side of the molecule. The optimization was performed with the Argus Lab program [16-19] Austin Model 1(AM1) in which the inclusion of enhanced and correlation function along with the polarization functions on heavier atoms and hydrogen's becomes correctly to report the nature of LUMO and other higher unoccupied orbitals. These serve as channels for electron tunnelling from acceptor to donor in an applied bias voltage. The electron transfer mechanism in the molecular system largely depends up on the spatial location [20] as well as spatial orientation of the frontier molecular orbitals. The molecular orbitals can be visualized using Argus Labs program. From the orbital HOMO, LUMO energy values, the potential drop (PD) across the molecule is calculated. This allows one to estimate if the system works like a rectifier when a suitable voltage applied to the molecule. At a suitable bias voltage the electron is loaded to the acceptor side (LUMO) and then it tunnels to the donor side (HOMO). This occurs when the rectifier molecule is in an instantaneous negatively-charged state.
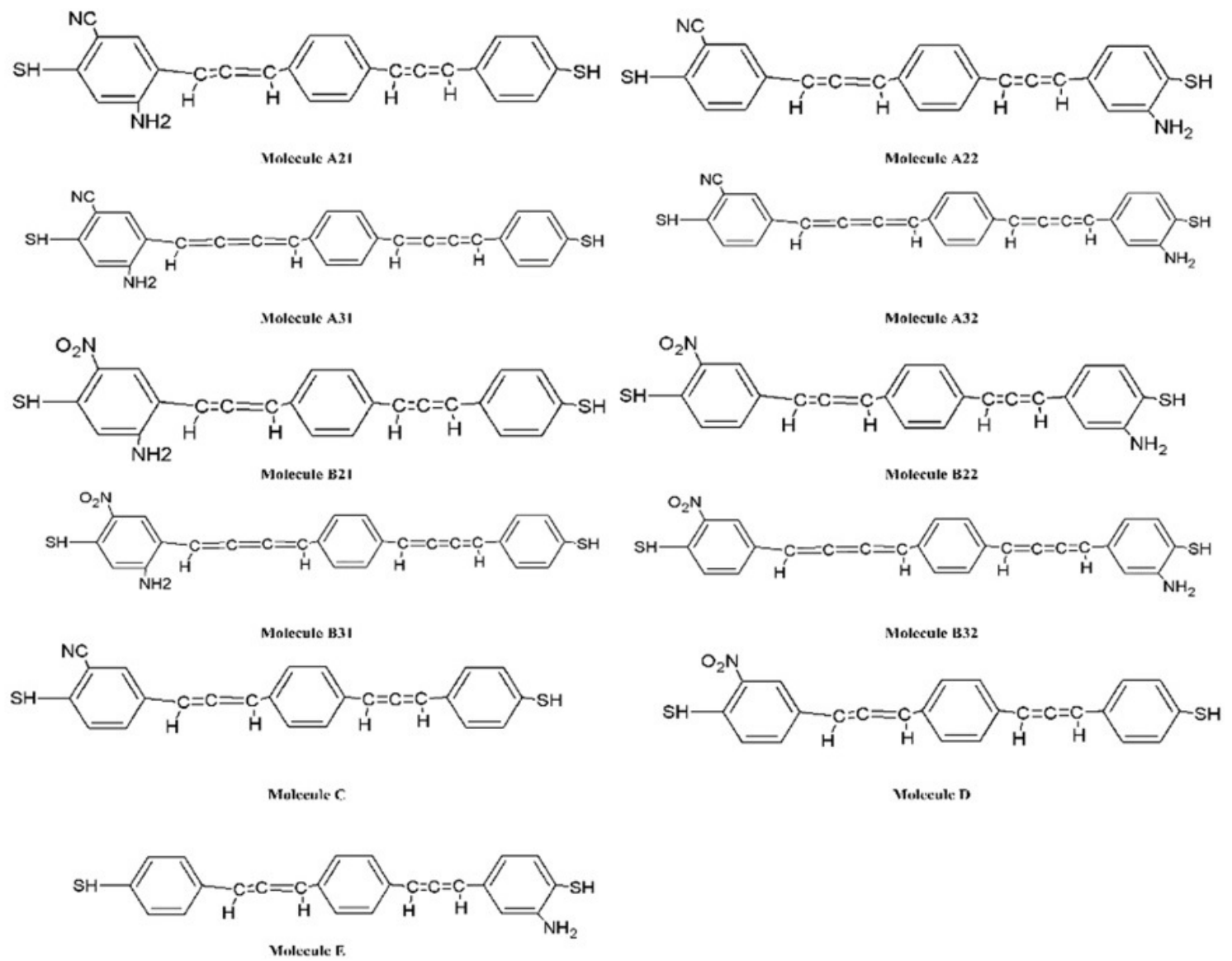

FIG. 1. Structure of donor $\pi$ acceptor nanowire which consists of a unimolecular bridge 
The molecular structures for DBA molecules A21, A22, A31, A32 were designed using $\mathrm{CN}$ and $\mathrm{NH}_{2}$ molecules with same position of benzene ring and opposite sides of the benzene position having odd and even number of separation bonds, while structures B21, B22, B31, B32 structures with $\mathrm{NO}_{2}$ and $\mathrm{NH}_{2}$ molecules in addition to that C, D, E structures are shown in Fig. 1. All Structures' HOMO and LUMO energy gaps were optimized and calculated via parametric method 3 in Argus lab [20].

Above listed molecules the DBA remains almost linear with small difference of linearity. The rigid linear construction of the DBA present in these molecules may be assigned to the involvement of the sp-carbon atoms in the bonding which is one the essential measures for device applications [27].

\section{Results and discussions}

\subsection{SP orbital energy levels}

The property and relation of these molecules A, B, C, D and E were carried out using AM1 optimization on individual Donor and acceptor heterocycles, and all were optimized with same methodology. Table 1 displays the HOMO and LUMO energies HLG and potential drop across the molecules calculated using equation 1:

$$
\mathrm{HLG}=\mathrm{E}_{H O M O}-\mathrm{E}_{L U M O},
$$

where $\mathrm{E}_{H O M O}$ and $\mathrm{E}_{L U M O}$ energies of $\mathrm{HOMO}$ and LUMO respectively.

Figure 2 shows the orbital energy diagram from HOMO and LUMO levels of different structures connected $\mathrm{CN}, \mathrm{NO}_{2}$ and $\mathrm{NH}_{2}$ molecular bridge at the same time in bridge energy levels observed with only acceptor molecule and donor molecule. Observation of table 1 results in the $n=2$ carbon atom chains separated with 2 sp hybridization and observe Fig. 1 A21,A22,B21 AND B22 where the LUMO and HOMO are still distributed over almost the entire molecule. The case changes radically $n=3$ two insulating groups move by $3.82 \mathrm{eV}, 4.57 \mathrm{eV}$, so that direct overlap between the sulfur and the LUMO and HOMO do not distribute from left to right. From this observation, rectification is obtained for sp chains greater than or equal to 2 .

TABLE 1. Orbital Energies of HOMO, LUMO, LUMO+1 for different molecules

\begin{tabular}{|c|c|c|c|c|c|c|c|c|}
\hline $\begin{array}{c}\text { Donor- } \\
\text { Acceptor }\end{array}$ & $\begin{array}{l}\text { Modal } \\
\text { Name }\end{array}$ & $\begin{array}{l}\text { HOMO } \\
\text { (a.u) }\end{array}$ & $\begin{array}{c}\text { LUMO } \\
\text { (a.u) }\end{array}$ & $\begin{array}{c}\text { LUMO+1 } \\
\text { (a.u) }\end{array}$ & $\begin{array}{c}\text { HLG\# } \\
\text { (a.u) }\end{array}$ & $\begin{array}{l}\text { HLG } \\
(\mathrm{eV})\end{array}$ & $\begin{array}{l}\text { PD }^{\$} \\
\text { (a.u) }\end{array}$ & $\begin{array}{l}\text { PD } \\
(\mathrm{eV})\end{array}$ \\
\hline \multirow{2}{*}{$\mathrm{CN}-\mathrm{NH}_{2}$} & A21 & 0.0283 & -0.1589 & -0.2368 & 0.1872 & 5.0940 & -0.0779 & 2.1198 \\
\hline & $\mathrm{A} 22$ & 0.025 & -0.1665 & -0.2389 & 0.1915 & 5.2110 & -0.0724 & 1.9701 \\
\hline \multirow{2}{*}{$\mathrm{CN}-\mathrm{NH}_{2}$} & A31 & -0.0431 & -0.1836 & -0.2484 & 0.1405 & 3.8232 & -0.0648 & 1.7633 \\
\hline & A32 & -0.0121 & -0.1801 & -0.2374 & 0.168 & 4.5715 & -0.0573 & 1.5592 \\
\hline \multirow{2}{*}{$\mathrm{NO}_{2}-\mathrm{NH}_{2}$} & B21 & 0.01313 & -0.182 & -0.2456 & 0.1951 & 5.3098 & -0.0636 & 1.7306 \\
\hline & B22 & -0.0055 & -0.1834 & -0.2462 & 0.1779 & 4.8409 & -0.0628 & 1.7089 \\
\hline \multirow{2}{*}{$\mathrm{NO}_{2}-\mathrm{NH}_{2}$} & B31 & 0.0198 & -0.2299 & -0.2582 & 0.2497 & 6.7947 & -0.0283 & 0.7701 \\
\hline & B32 & 0.0109 & -0.2373 & -0.2448 & 0.2482 & 6.7539 & -0.0075 & 0.2041 \\
\hline $\mathrm{CN}$ & $\mathrm{C}$ & 0.021 & -0.1707 & -0.2464 & 0.1917 & 5.2164 & -0.0757 & 2.0599 \\
\hline $\mathrm{NO}_{2}$ & $\mathrm{D}$ & 0.0076 & -0.1872 & -0.2507 & 0.1948 & 5.3008 & -0.0635 & 1.7279 \\
\hline $\mathrm{NH}_{2}$ & E & 0.038 & -0.1451 & -0.2253 & 0.1831 & 4.9824 & -0.0802 & 2.1824 \\
\hline
\end{tabular}

\subsection{The shift of molecular orbital \& transport analysis}

The DBA molecule is connected between the electrodes i.e., donor is connected to the positive terminal and acceptor is connected to negative terminal and a suitable voltage is applied one electron will be entered to the molecule from the negative side. The enrolled electron inside the bridge will be placed in the LUMO of the molecule localized on the acceptor side. Then this electron tunnels in through the flexible bridge to the donor side and finally escapes to the anode through the unoccupied molecular orbital located on the donor side of the molecule. In a rectifying molecular bridge, this tunnelling process is largely controlled by the unoccupied orbitals (LUMO's are used for the electrical conduction inside the molecule). 


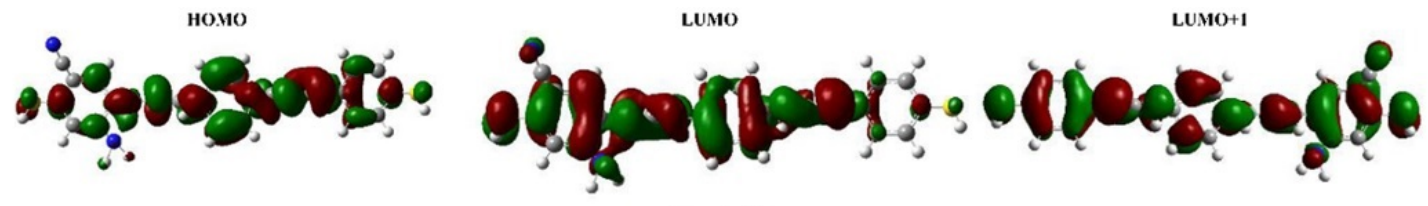

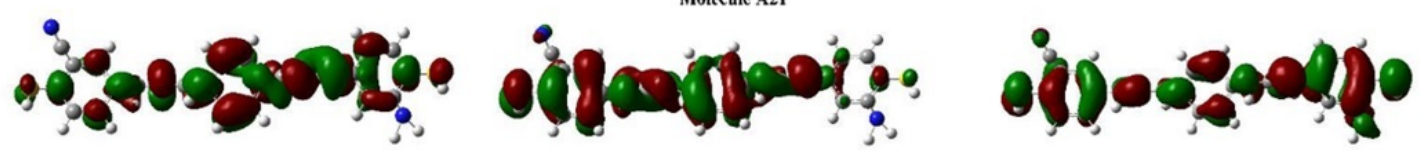

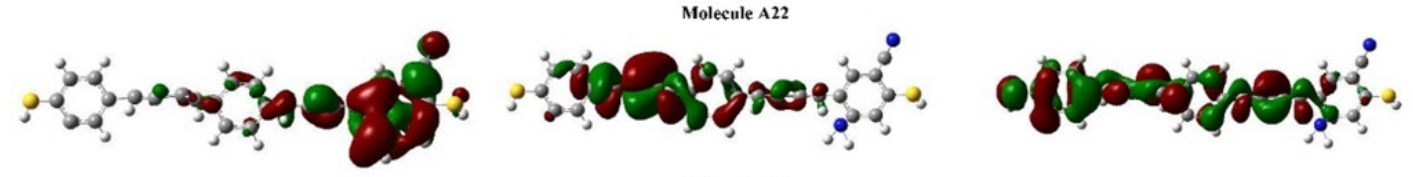

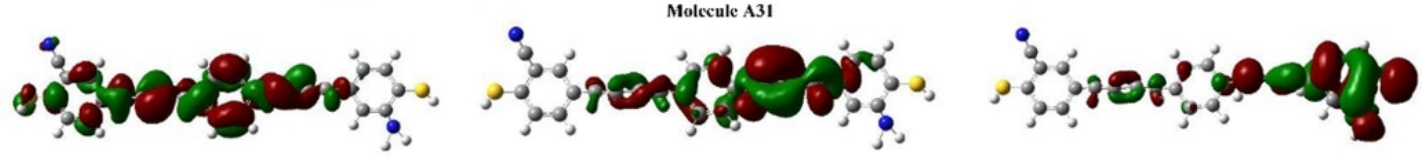

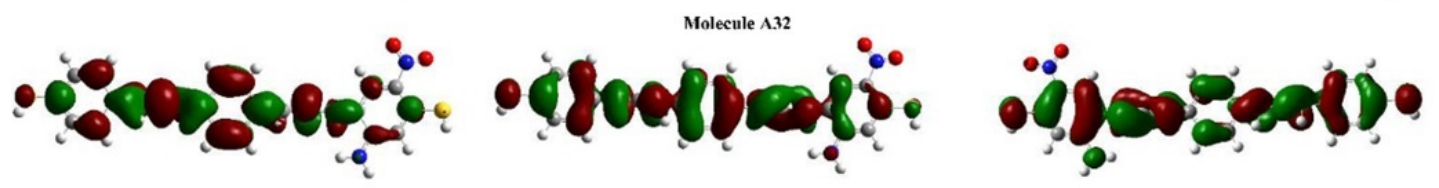

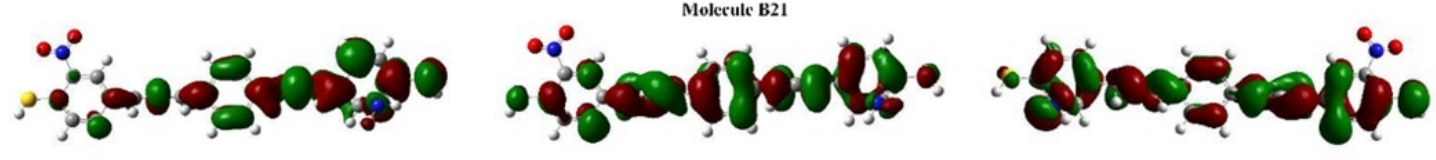

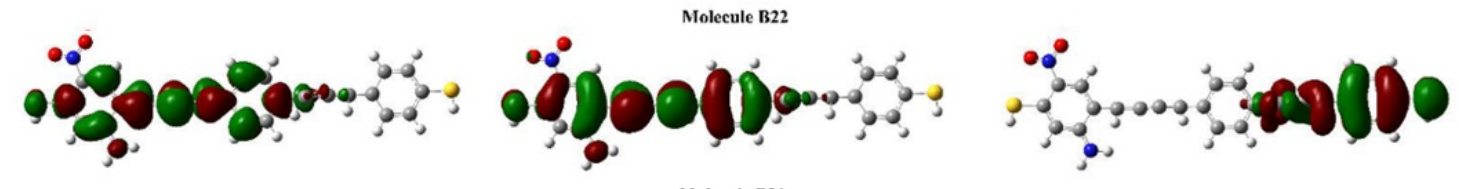

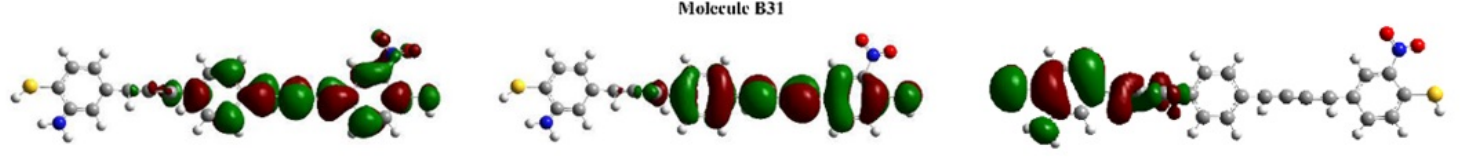
\%

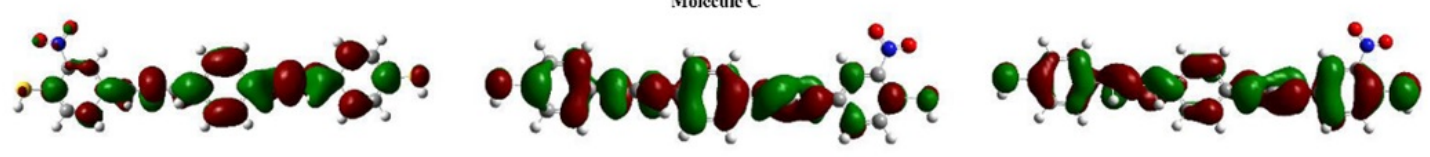

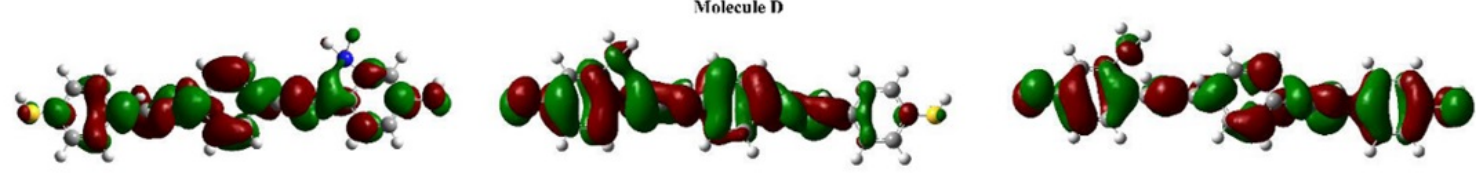


The abovediscussed bridge operation for electron transfer in both the reverse and forward directions of bias applied to the molecule in this study is described as follows: in the forward direction the applied positive energy required for raising is greater than the Fermi energy of the electron in the occupied level of the sulfur contact on the acceptor side, closely as high as the energy of the LUMO, which is located acceptor part of the molecule. The inserted electron from the sulfur contact to the acceptor part of the molecule can travel through the central bridge to the unoccupied molecular orbital present in the donor of the molecule and finally escape into the sulfur contact which is connected in the donor part of bridge. In a reverse bias condition for electron transfer from the donor to the acceptor part of the molecule through the central bridge, the applied voltage bias must be sufficient more to increase the Fermi energy of the sulfur contact on the donor side of the molecule, due to that it would match with the energy of the LUMO+1 situated on the donor part of the molecule. To maintain current flow through the molecule sufficient surplus voltage is given in the forward direction compared to the reverse direction bias voltage because that electron jumps from the LUMO+1 to the LUMO level.

TABLE 2. The calculated Muliken charge ranges, Total energy and dipole moment of molecular bridge

\begin{tabular}{|c|c|c|c|}
\hline $\begin{array}{c}\text { Modal } \\
\text { Name }\end{array}$ & $\begin{array}{c}\text { Muliken Charge } \\
\text { Range (eV) }\end{array}$ & $\begin{array}{c}\text { Total } \\
\text { Energy (Kcal/Mol.) }\end{array}$ & $\begin{array}{c}\text { Dipole } \\
\text { Moment (Debye) }\end{array}$ \\
\hline A21 & -1.041 to 1.041 & -1849.0303 & 16.3230 \\
\hline A22 & -0.828 to 0.828 & -1848.7505 & 18.0507 \\
\hline A31 & -0.632 to 0.632 & -1923.8167 & 12.4588 \\
\hline A32 & -0.826 to 0.826 & -1924.0890 & 13.7230 \\
\hline B21 & -1.048 to 1.048 & -1960.9553 & 11.6610 \\
\hline B22 & -0.785 to 0.785 & -1960.5074 & 15.0299 \\
\hline B31 & -1.044 to 1.044 & -2036.1977 & 12.1986 \\
\hline B32 & -1.099 to 1.099 & -2036.0312 & 14.6837 \\
\hline C & -0.630 to 0.630 & -1794.1484 & 15.7409 \\
\hline D & -0.535 to 0.535 & -1906.3258 & 10.7947 \\
\hline E & -1.045 to 1.045 & -1757.5900 & 9.0366 \\
\hline
\end{tabular}

Using population analyses, and as an effect of the rectifying properties of the molecular systems, the electron is transmitted through the molecule. The spatial position of the molecular orbitals plays an important role in accounting for electron transport in the molecule. The Potential Drop (PD) across the molecule gives information about the effectiveness of the DBA to behave as a rectifier when voltage is applied to the molecule. The PD value obtained from orbital energy values is as follows:

$$
\text { Potential Drop } \quad(P D)=\Delta E_{L U M O}=E_{L U M O+K}-E_{L U M O} ; \quad k=0,1,2,
$$

where $E_{L U M O}$ is the orbital energy localized on the acceptor side and $E_{L U M O+K}$ is the orbital energy of the next unoccupied orbital (here it is LUMO+1), which is localized on the donor side of the molecule. The approximate PD across the proposed molecular rectifiers have been calculated using above equation 2 and listed in Table 2. The spatial location and orientations of frontier molecular orbitals are shown in Fig. 2 for molecules A,B,C,D,E, in which the two ends of the cumulenic bridge acceptor and donor are connected via even and odd number double bonds.

\section{Conclusion}

The electron transport properties of molecular bridges containing amine group and nitro groups with odd and even number of double bonds and also varying position of the donor $\left(\mathrm{NH}_{2}\right)$ and acceptor $\left(\mathrm{NO}_{2}, \mathrm{CN}\right)$ molecules energies were calculated using Extended Hückel and Parametric Methods. From our observations of energy HOMO, LUMO and HLG bridges having an odd number double bonds showed molecular conductance and should have utility as a molecular wire, heterocyclic with even number of double bonds work as a rectifying diode in a electronic circuits. Therefore, a pure static treatment based on EHT without calculating the current could be an alternative approach from the NEGF methods in studying or designing the novel molecular electronic devices. 


\section{Acknowledgement}

The authors would like to thank Dr. K. Bhanuprakash, Chief Scientist, CSIR-IICT, Hyderabad, for encouragement and support throughout the period of this work and ECE Department, JNTUA, Ananthapuramu, Gudlavalleru Engineering College, Gudlavalleru, provided the computing facilities available to carry out the simulation work.

\section{References}

[1] Aviram A., Ratner M.A. Molecular rectifiers. Chemical Physics Letter, 1974, 29(2), P. 277-283.

[2] Datta S. Nanoscale device modeling: the Green's function method. Super lattices and Microstructures, 2000, 28(4), P. 253-278.

[3] Datta S. Electronic Transport in Mesoscopic Systems Cambridge University Press. New York, 1996,200 p.

[4] Pradan M.R., Rajan E.G. A system engineering Approach to Molecular Electronics. International Journal of Computer applications, 2010, 3(8), P. 14-23.

[5] Fobelets K., Ding P., et al. Electrical Transport in Polymer-Covered Silicon Nanowires. IEEE Transactions on Nanotechnology, 2012, $11(4)$, P. 661-665.

[6] Jin-woo kim, Jeong-Hwan Kim, Russell Deaton. Programmable Construction of Nanostructures. IEEE Nanotechnology Magazine, 2012, 2(3), P. 19-23.

[7] Xia Y., Chu Z., Hung W.N.N. An Integrated Optimization Approach for Nano hybrid Circuit Cell Mapping. IEEE Transactions on Nanotechnology, 2011, 10(6) P. 1275-1284.

[8] Damle P.S., Ghosh A.W., Datta S. Unified description of molecular conduction: From molecules to metallic wires. Physics Review $B, 2001,64(20)$, P. 201403-1-201403-4

[9] Datta S., Tian W., et al. Current-Voltage Characteristics of Self-Assembled Monolayers by Scanning Tunneling Microscopy. Physics Review Letters, 1997, 79(13), P. 2530-2533.

[10] Xue Y., Ratner M.A. Microscopic study of electrical transport through individual molecules with metallic contacts. I. Band lineup, voltage drop and high field transport. Physics Review B, 2003, 68, P. 115406

[11] Song H., Kim Y., et al. Observation of molecular orbital gating Nature Letters, 2009, 462(24), P. 1039-1043.

[12] Pecchia A., Di Carlo A., et al. Incoherent electron-phonon scattering in octanethiols. Nano Letters, 2004, 4(11), P. 2109-2114.

[13] Wan C.C., Mozos J.-L., et al. Quantized conductance of Si atomic wires. Physical Review B, 1997, 56(8), P. R4351-R4354.

[14] Wang Z., Kadohira T., Tada T., Watanabe S. Nonequilibrium Quantum Transport Properties of a Silver Atomic Switch. Nano Letters, 2007, 7(9), P. 2688-2692.

[15] Xu Y., Shi X., et al. Conductance oscillation and quantization in monatomic AI wires. Journal of Physics: Condensed Matter, 2007, 19(5), P. 056010.

[16] Parlato F.R., Barra L., et al. Single molecule break junctions based on a perylene diimide cyano functionalized derivative. Nanoscale Research Letter, 2015, 10, P. 305.

[17] Yu Zhang L., Friesner R.A. Ab initio calculation of electronic coupling in the photosynthetic reaction center. Proceedings of the National Academy of Sciences of the United States of America, 1998, 95, P. 13603-13605.

[18] Thompson M.A., Glendening E.D., Feller D. The Nature of K+/Crown Ether Interactions: A Hybrid Quantum MechanicalMolecular Mechanical Study. The Journal of Physical Chemistry, 1994, 98(41), P. 10465-10476.

[19] Thompson M.A., Schenter G.K. Excited States of the Bacteriochlorophyll b Dimer of Rhodopseudomonas viridis: A QM/MM Study of the Photosynthetic Reaction Center That Includes MM Polarization. The Journal of Physical Chemistry, 1995, 99, P. 6374-6386.

[20] Thompson M.A. QM/MMpol: A Consistent Model for Solute/Solvent Polarization. Application to the Aqueous Solvation and Spectroscopy of Formaldehyde, Acetaldehyde, and Acetone. The Journal of Physical Chemistry, 1996, 100, P. 14492-14507.

[21] Dewar M.J.S., Zoebisch E., et al. Development and use of quantum mechanical molecular models. 76. AM1: a new general purpose quantum mechanical molecular model. Journal of the American Chemical Society, 1985, 107(13), P. 3902-3909.

[22] Joachim C., Gimzewski J.K., Aviram A. Electronics using hybrid-molecular and mono-molecular devices Nature. Macmillan Magazines Limited, 2000, 408, P. 541-548.

[23] Tian W., Datta S., et al. Conductance spectra of molecular wires. The Journal of Chemical Physics, 1998, 109(7) P. 2874-2882.

[24] Xue Y.Q., Datta S., Ratner M.A. First-principles based matrix Green's function approach to molecular electronic devices: general formalism. Chemical Physics, 2002, 281(2), P. 151-170.

[25] Yaliraki S.N., Roitberg A.E., et al. The injecting energy at molecule/metal interfaces: Implications for conductance of molecular junctions from an ab initio molecular description. The Journal of chemical physics, 1999, 111(15), P. 6997-7002.

[26] Ghosh A.W., Data S. Molecular conduction: paradigms and possibilities. Journal of Computational electronics, 2002, 1(4), P. 515-525.

[27] Amgad Ahmed Ali Abdul Manaf Hashim. Density functional theory study of atomic layer deposition of zinc oxide on grapheme. Nanoscale Research Letters, 2015, 10, P. 299. 